\title{
The expression of NMDA receptor and pCREB in the visual cortex of monocularly-deprived rats
}

\author{
XIAONAN SUN ${ }^{1,2}$ and JINSONG ZHANG ${ }^{1}$ \\ ${ }^{1}$ The Fourth Affiliated Hospital of China Medical University, Shenyang, Liaoning 110005; \\ ${ }^{2}$ The Fourth People's Hospital of Shenyang, Shenyang, Liaoning 110031, P.R. China
}

Received August 21, 2012; Accepted January 7, 2013

DOI: $10.3892 / \mathrm{mmr} .2013 .1304$

\begin{abstract}
N-methyl-D-aspartate receptor subunit 1 (NMDAR1) and cAMP response element binding protein (CREB) play a role in vision plasticity. However, the correlation between their expression and vision plasticity is not clear. The present study aimed to examine the expression of NMDAR1 and phosphorylated CREB (pCREB) in the visual cortex of monocularly-deprived (MD) rats in the developmental phase. Eighty healthy Sprague Dawley rats were randomly divided into 4 groups $(\mathrm{n}=20)$; normal, MD, KN-93 (MD rats treated with KN-93, a calmodulin kinase IV inhibitor) and saline (MD rats treated with saline as control). All rats were reared for 45 days in a naturally lit environment. The expression of NMDAR 1 and pCREB in the hibateral visual cortex of rats from each group was detected by immunohistochemistry and western blot analysis. The results demonstrate that, compared with the normal group, the expression of NMDAR1 and pCREB was increased in deprived and decreased in the non-deprived-side visual cortex in MD. Compared with the saline group, no significant difference was identified in NMDAR1 expression while pCREB expression was decreased in the deprived-side visual cortex in KN-93. No significant difference in the expression of NMDAR1 and pCREB in the non-deprived-side visual cortex between the KN-93 and saline groups was observed. The results indicate that NMDAR1 and CREB are involved in the ocular dominance forming process and play a role in vision plasticity.
\end{abstract}

\section{Introduction}

The N-methyl-D-aspartate receptor (NMDAR) is a subtype of ion-type glutamic acid receptor and is associated with a variety of physiological processes, including excitatory synaptic transmission, learning and memory and neural development.

Correspondence to: Dr Jinsong Zhang, The Fourth Affiliated Hospital of China Medical University, 102 Nanqi Road, Shenyang 110005, P.R. China

E-mail: zhangjinsongcmu@126.com

Key words: NMDA receptor, CREB, monocular deprivation, calmodulin kinase IV, vision
In particular, NMDA is important in the development of the nervous system via the regulation of neuronal migration and synaptic plasticity (1). NMDAR is a membrane ionophorous protein composed of NR1, the functional subunit constituting the ion channel and NR2, the regulatory subunit (2). NMDAR is a voltage and ligand doubly gated channel and $\mathrm{Mg}^{2+}$ not only blocks the NMDA channel in a voltage-dependent manner but also potentiates NMDA-induced responses at positive membrane potentials. Magnesium glycinate and magnesium taurinate treatment have been utilized to provide rapid recovery from depression (3). When the postsynaptic membrane depolarizes, channels open and large quantities of $\mathrm{Ca}^{2+}$ enter the cells to function as second messengers to activate calmodulin kinase IV (CaMK IV), which then phosphorylates cAMP response element binding protein (CREB) on Ser133 in the active site. Following phosphorylation, CREB interacts with CBP (CREB-binding protein) to activate transcription of downstream genes.

A number of previous studies have identified a role for CREB in vision plasticity $(4,5)$. However, the correlation between NMDAR and CREB expression and vision plasticity remains unclear. In the present study, expression levels of NMDAR and CREB were detected in the visual cortex of monocularly-deprived (MD) animal models. Results of the present study are likely to provide insight into the molecular mechanism of vision plasticity.

\section{Materials and methods}

Animal groups. Eighty Sprague Dawley pedo-rats (2 weeks old) were obtained from the animal department of the China Medical University (Shenyang, China) and divided into 4 groups $(n=20)$. The normal group received no treatment. The MD group was anesthetized by peritoneal injection of 5\% chloral hydrate $(3 \mathrm{ml} / \mathrm{kg})$. Following this, the pelage surrounding the palpebral margin was removed, the area was sterilized with iodophors and the superior and inferior palpebral margins were cut $1-1.5 \mathrm{~mm}$ from the inner to external canthus. Experimental eyelids were sutured closed using the mattress suture with 6-0 suture silk to adhere the superior and inferior eyelids to form MD (right eye-deprived). The saline group consisted of MD rats injected with saline into the archae-visual cortex ocellanae region. The KN-93 group consisted of MD rats injected with the calmodulin kinase IV inhibitor, KN-93 (dissolved in saline as $1 \mu \mathrm{g} / 4 \mu \mathrm{l}$ ) into archae-visual cortex ocellanae regions. Rats 
Table I. NMDAR1 and pCREB protein expression levels in non-deprived and deprived-side visual cortex in MD and normal groups (mean $\pm \mathrm{SD})$.

\begin{tabular}{|c|c|c|c|c|c|}
\hline \multirow[b]{2}{*}{ Group } & \multirow[b]{2}{*}{$\mathrm{n}$} & \multicolumn{2}{|c|}{ NMDAR1 } & \multicolumn{2}{|c|}{ pCREB } \\
\hline & & Non-deprived & Deprived & Non-deprived & Deprived \\
\hline MD & 10 & $0.132 \pm 0.01$ & $0.498 \pm 0.05$ & $0.309 \pm 0.05$ & $0.652 \pm 0.03$ \\
\hline Normal & 10 & $0.249 \pm 0.03$ & $0.277 \pm 0.03$ & $0.391 \pm 0.03$ & $0.433 \pm 0.03$ \\
\hline $\mathrm{t}$ & & 11.700 & 11.985 & 4.447 & 16.323 \\
\hline P-value & & $<0.05$ & $<0.05$ & $<0.05$ & $<0.05$ \\
\hline
\end{tabular}

NMDAR1, N-methyl-D-aspartate receptor subunit 1; pCREB, phosphorylated cAMP response element binding protein; MD, monocularly-deprived.
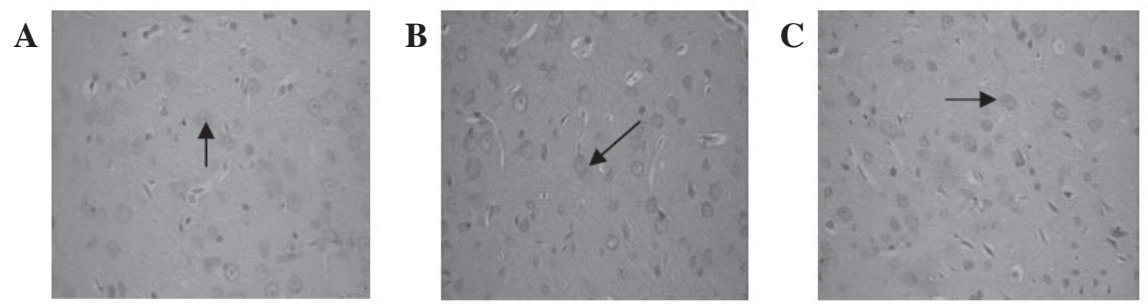

Figure 1. Immunohistochemical staining of NMDAR1 in normal and MD rats. Monocular deprivation led to (A) reduced staining of NMDAR1 in the non-deprived-side visual cortex of rats and (B) increased staining of NMDAR1 in the deprived-side visual cortex of rats. (C) NMDAR1 staining in visual cortex of rats in normal group. Magnification, x400.

in each group were reared in the same naturally lit environment for 45 days. The experimental protocol was approved by the Ethics Committee of the China Medical University.

Immunohistochemistry. Hibateral visual cortices were removed from 10 rats in each group, fixed with $4 \%$ paraformaldehyde and embedded in mineral wax. Following this, slides were treated with dimethyl benzene and subjected to hydration with sequential ethanol, washed with $0.01 \mathrm{~mol} / \mathrm{l} \mathrm{PBS}$ (3 times for $5 \mathrm{~min}$ ), then incubated with $0.5 \%$ parenzyme for $30 \mathrm{~min}$ at $37^{\circ} \mathrm{C}$. Next, slides were washed with PBS (as described), incubated with $3 \% \mathrm{H}_{2} \mathrm{O}_{2}$ for $15 \mathrm{~min}$ at room temperature to block endogenous peroxidase, washed with PBS (as described) and incubated with normal goat serum for $15 \mathrm{~min}$ at room temperature. Slides were then incubated overnight at $4^{\circ} \mathrm{C}$ with primary antibody against NMDAR1 (1:1,000; Pharmigen, San Diego, CA, USA) and pCREB (1:50, Cell Signaling, Danvers, MA, USA), followed by incubation with HRP-labeled secondary antibody for $30 \mathrm{~min}$ at $37^{\circ} \mathrm{C}$ and $\mathrm{DAB}$ chromogenic reaction. Slides were counterstained with hematoxylin and observed under a microscope.

Western blot analysis. Hibateral visual cortices were removed from 10 rats of each group and lysed in RIPA buffer by homogenization on ice. Lysates were collected following centrifugation at $12,000 \mathrm{rpm}$ for $10 \mathrm{~min}$ at $4^{\circ} \mathrm{C}$. Protein levels were quantified using the bicinchoninic acid method. Equivalent amounts of protein ( $30 \mu \mathrm{g} / \mathrm{lane})$ were separated by $10 \%$ sodium dodecyl sulfate-polyacrylamide gel electrophoresis and transferred to PVDF membranes. Membranes were blocked in PBS containing 5\% non-fat dry milk (w/v) for $4 \mathrm{~h}$ at $37^{\circ} \mathrm{C}$ and then incubated with primary antibodies against NMDAR1 $(1: 1,000)$ and pCREB $(1: 50)$ overnight at $4^{\circ} \mathrm{C}$. Membranes were then incubated with HRP-conjugated secondary antibodies at room temperature for $1 \mathrm{~h}$, developed using enhanced chemiluminescence reagent and exposed to X-ray film in a dark room. $\beta$-actin was used as loading control. Gelpro32 gel image analysis software was used to perform densitometric analysis of the blots and the ratio of values obtained for NMDAR1 and pCREB to $\beta$-actin in each group was considered to represent the relative amount of target protein for each sample.

Statistical analysis. Data are expressed as the mean \pm SD. Statistical analysis was performed using the SPSS software 13.0 (SPSS Inc., Chicago, IL, USA). P $<0.05$ was considered to indicate a statistically significant difference.

\section{Results}

Comparison of NMDARI and pCREB levels in the visual cortex of rats in $M D$ and normal groups. Immunohistochemical staining identified that compared with normal rats, the number of NMDAR1-positive cells was increased in the deprived but decreased in the non-deprived-side visual cortex in MD rats (Fig. 1). Similarly, the number of pCREB-positive cells was increased in the deprived- but decreased in the non-deprivedside visual cortex in MD, compared with the normal rat group (Fig. 2).

Western blot analysis demonstrated that relative protein expression of NMDAR1 was significantly higher in the deprived-side visual cortex of MD $(0.498 \pm 0.05)$ compared with 
Table II. NMDAR1 and pCREB protein expression levels in non-deprived and deprived-side visual cortex in KN-93 and saline groups (mean $\pm \mathrm{SD})$.

\begin{tabular}{|c|c|c|c|c|c|}
\hline \multirow[b]{2}{*}{ Group } & \multirow[b]{2}{*}{$\mathrm{n}$} & \multicolumn{2}{|c|}{ NMDAR1 } & \multicolumn{2}{|c|}{ pCREB } \\
\hline & & Non-deprived & Deprived & Non-deprived & Deprived \\
\hline KN-93 & 10 & $0.1457 \pm 0.02$ & $0.501 \pm 0.05$ & $0.326 \pm 0.05$ & $0.331 \pm 0.03$ \\
\hline NS & 10 & $0.1457 \pm 0.02$ & $0.485 \pm 0.08$ & $0.315 \pm 0.07$ & $0.666 \pm 0.05$ \\
\hline $\mathrm{t}$ & & 0.0175 & 0.536 & 0.404 & 18.168 \\
\hline P-value & & $>0.05$ & $>0.05$ & $>0.05$ & $<0.05$ \\
\hline
\end{tabular}

NMDAR1, N-methyl-D-aspartate receptor subunit 1; pCREB, phosphorylated cAMP response element binding protein.

A

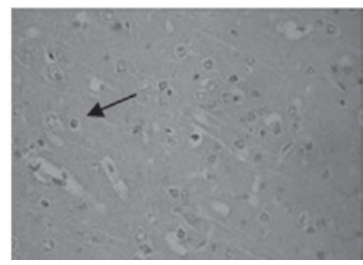

B

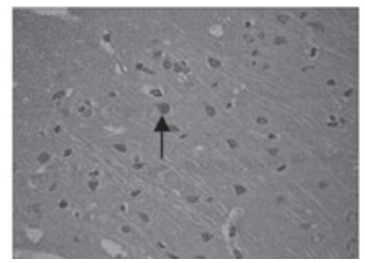

C

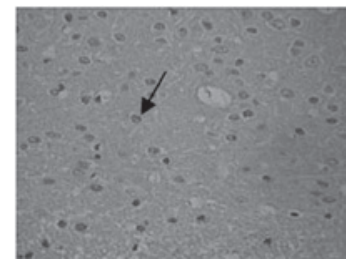

Figure 2. Immunohistochemical staining of pCREB in normal and MD rats. MD led to (A) reduced staining of pCREB in the non-deprived-side visual cortex of rats and (B) increased staining of pCREB in the deprived-side visual cortex of rats. (C) pCREB staining in the visual cortex of rats in normal group. Magnification, x400. pCREB, phosphorylated cAMP response element binding protein; MD, monocularly-deprived.

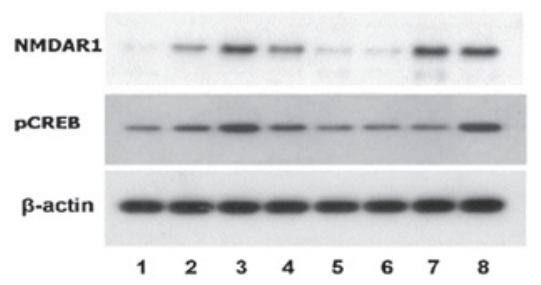

Figure 3. Western blot analysis of NMDAR1 and pCREB levels in the visual cortex of rat groups. 1 , non-deprived side in $\mathrm{MD} ; 2$, non-deprived side in normal; 3, deprived side in MD; 4, deprived side in normal; 5, non-deprived side in $\mathrm{KN}-93$; 6, non-deprived side in NS; 7, deprived side in KN-93; and 8 , deprived side in NS groups. $\beta$-actin served as a loading control. Representative blots from three independent experiments are presented. No monocular eyelid suture in normal group was performed, therefore the non-deprived side of normal was in homonymy with the non-deprived side of MD. NMDAR1, $\mathrm{N}$-methyl-D-aspartate receptor subunit 1; pCREB, phosphorylated cAMP response element binding protein; $\mathrm{MD}$, monocularly-deprived.
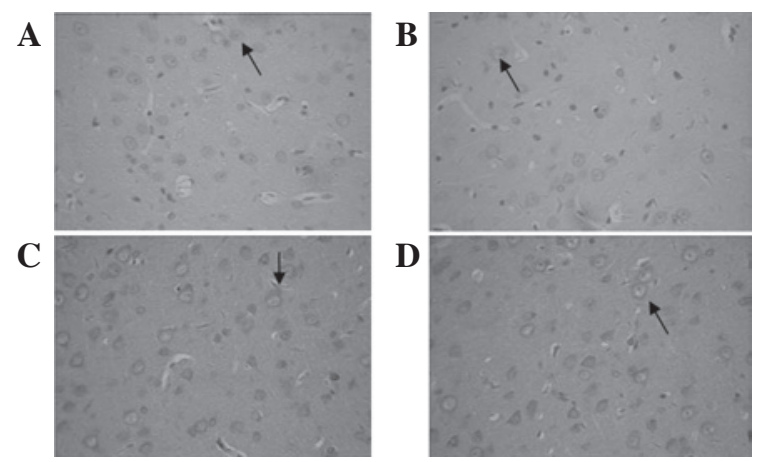

Figure 4. Immunohistochemical staining of NMDAR1 in KN-93 and saline groups. NMDAR1 staining in the non-deprive-side visual cortex of MD rats following (A) KN-93 and (B) saline treatments. NMDAR1 staining in the deprive-side visual cortex of MD rats following (C) KN-93 and (D) saline treatments. Magnification, x400. NMDAR1, N-methyl-D-aspartate receptor subunit 1. MD, monocularly-deprived. the normal group $(0.277 \pm 0.03$; Fig. $3 \mathrm{~A}$ and Table $\mathrm{I}, \mathrm{t}=11.985$, $\mathrm{P}<0.05)$. Similarly, pCREB protein levels were observed to be significantly higher in the deprived-side visual cortex of MD $(0.652 \pm 0.03)$ compared with the normal group $(0.433 \pm 0.03$; Fig. 3B and Table I, $\mathrm{t}=16.323, \mathrm{P}<0.05)$. However, NMDAR1 protein expression was found to be significantly lower in the non-deprived-side visual cortex of MD $(0.132 \pm 0.01)$ compared with the normal group $(0.249 \pm 0.03$; Fig. $3 \mathrm{~A}$, Table $\mathrm{I}$, $\mathrm{t}=11.700$, $\mathrm{P}<0.05)$ and $\mathrm{pCREB}$ protein expression was significantly lower in the non-deprived-side visual cortex of MD $(0.309 \pm 0.05)$ compared with the normal group $(0.391 \pm 0.03$; Fig. 3B, Table I, $\mathrm{t}=4.447, \mathrm{P}<0.05)$. Results are consistent with immunohistochemical analysis.

Comparison of NMDAR1 and pCREB levels in the visual cortex of rats between $K N-93$ and saline groups. Immunohistochemical staining revealed that the number of NMDAR1-positive cells was not significantly different in the deprived- and non-deprived-side visual cortices between the KN-93 and saline groups (Fig. 4). However, the number of pCREB-positive cells was reduced in the deprived-side visual cortex of KN-93 compared with that of the saline group (Fig. 5). In addition, the number of pCREB-positive cells was not found to be significantly different in the non-deprived-side visual cortex of KN-93 compared with the saline group (Fig. 5).

Western blot analysis demonstrated that NMDAR1 protein levels were not significantly different between the deprived-side visual cortex of KN-93 $(0.485 \pm 0.08)$ and the NS group (0.498 \pm 0.05 ; Fig. 3A, Table II, $\mathrm{t}=0.536, \mathrm{P}>0.05)$. However, pCREB protein levels were observed to be significantly lower in the deprived-side visual cortex of KN-93 $(0.331 \pm 0.03)$ compared with the saline group $(0.666 \pm 0.05$; Fig. 3B, Table II, $\mathrm{t}=18.168, \mathrm{P}<0.05)$. However, NMDAR1 

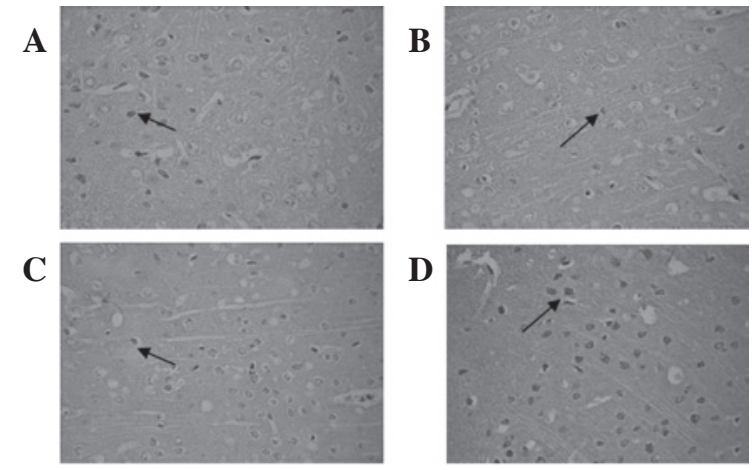

Figure 5. Immunohistochemical staining of pCREB in KN-93 and saline groups. pCREB staining in the non-deprived-side visual cortex of MD rats following (A) KN-93 and (B) saline treatments. pCREB staining in the deprive-side visual cortex of MO rats following (C) KN-93 and (D) saline treatments. Magnification, x400. pCREB, phosphorylated cAMP response element binding protein; MD, monocularly-deprived.

protein expression was significantly lower $(0.132 \pm 0.01)$ in the non-deprived-side visual cortex of MD compared with the normal group $(0.249 \pm 0.03$; Fig. $3 \mathrm{~A}, \mathrm{t}=11.700, \mathrm{P}<0.05)$ and pCREB protein expression was observed to be significantly lower $(0.309 \pm 0.05)$ in the non-deprived-side visual cortex of MD rats compared with the normal group $(0.391 \pm 0.03$; Fig. 3B, $\mathrm{t}=4.447, \mathrm{P}<0.05)$.

In the non-deprived-side visual cortex, western blot analysis revealed that NMDAR 1 protein levels were $0.1457 \pm 0.02$ in KN-93 and $0.1459 \pm 0.03$ in saline groups. No statistically significant difference was identified between the groups (Fig. 3 and Table I, t=0.0175, P>0.05). Similarly, pCREB protein levels were $0.326 \pm 0.05$ in $\mathrm{KN}-93$ and $0.315 \pm 0.07$ in the saline groups. No statistically significant difference was found between the groups (Fig. 3 and Table II, $\mathrm{t}=0.404, \mathrm{P}>0.05$ ). Results are consistent with those of immunohistochemical analysis.

\section{Discussion}

CREB is a nuclear factor with important biological functions, including regulation of learning and memory $(6,7)$. The transcriptional activity of CREB mainly relies on the phosphorylation of Ser133, catalyzed by CaMK IV. NMDAR is an excitation pattern ionophorous protein residing in the postsynaptic membrane of neurons. NMDA induced $\mathrm{Ca}^{2+}$ overload is important for activation of the downstream cascades (8).

The NMDAR-CREB signaling cascade has been demonstrated to protect against extrasynaptic NMDAR-induced neuronal cell death and ischemic brain damage (9). However, the role of the NMDAR-CREB signaling cascade in vision plasticity remains unclear.

A number of studies have identified that NMDAR1 expression is altered in the visual cortex under various conditions (10-12). However, studies have yet to examine the expression and activation of CREB under these conditions and determine the correlation between NMDAR1 expression and CREB expression and activation. To the best of our knowledge, this is the first study to examine this correlation in MD rats. In addition, previous studies have only compared NMDAR1 expression between the deprived and non-deprived-side visual cortex of model rats. In the present study NMDAR1 expression was compared in the deprived or non-deprived side to that in corresponding sides of normal control rats, a more appropriate method to identify the competition mechanism of reciprocal growth and decline in the projection of binocular retinal ganglial cells to the visual cortex. Using immunohistochemical staining and western blot analysis, distinct alterations in NMDAR1 expression in the hibateral visual cortex were observed following monocular deprivation. NMDAR1 expression was reduced in the non-deprived-side visual cortex which received the projection of ganglion cells of the deprived side, but was increased in the deprived-side visual cortex which received the projection of ganglion cells of the non-deprived side.

A limited number of studies have examined the expression of pCREB during vision plasticity. In the present study, immunohistochemistry and western blot analysis revealed that pCREB levels were increased in the deprived and reduced in the non-deprived-side visual cortex compared with the normal group. Results demonstrate that altered pCREB expression correlates with NMDAR1 expression in the deprived and non-deprived-side visual cortex, indicating that the NMDAR-CREB cascade may play a role in the formation process of vision plasticity.

To test this hypothesis, cells were treated with KN-93, a specific inhibitor of CaMK IV which catalyzes the phosphorylation of CREB and promotes the activation of CREB target genes. The results of immunohistochemistry and western blot analysis found no significant difference in NMDAR1 expression in the visual cortex between the KN-93 and saline groups, indicating that inhibition of CaMK IV does not affect NMDAR1 expression, which lies upstream of the NMDAR-CREB cascade. However, as expected, pCREB levels in the deprived-side visual cortex in KN-93 were lower than those in the saline group. Collectively, results indicate that CaMK IV functions upstream of CREB but downstream of NMDAR to modulate vision plasticity.

In summary, results of the present study demonstrate that NMDAR1 and pCREB levels in the visual cortex of MD rats were higher in the deprived and lower in the non-deprived sides compared with the normal group, which implies that NMDAR1 and CREB are involved in the ocular dominance forming process during vision development. CaMK IV inhibitor reduced pCREB but not NMDAR1 protein levels in the deprived and non-deprived sides, demonstrating that the NMDAR-CaMK IV-CREB axis is associated with regulation of vision plasticity.

\section{Acknowledgements}

The authors thank Dr Shaoli Zhou and Dr Jiangyue Zhao for their assistance with this study.

\section{References}

1. Li F and Tsien JZ: Memory and the NMDA receptors. N Engl J Med 361: 302-303, 2009.

2. Paoletti P and Neyton J: NMDA receptor subunits: function and pharmacology. Curr Opin Pharmacol 7: 39-47, 2007.

3. Eby GA and Eby KL: Rapid recovery from major depression using magnesium treatment. Medical hypotheses 67: 362-370, 2006 . 
4. Mower AF, Liao DS, Nestler EJ, Neve RL and Ramoa AS cAMP/Ca ${ }^{2+}$ response element-binding protein function is essential for ocular dominance plasticity. J Neurosci 22: 2237-2245, 2002.

5. Pham TA, Graham SJ, Suzuki S, Barco A, Kandel ER, Gordon B and Lickey ME: A semi-persistent adult ocular dominance plasticity in visual cortex is stabilized by activated CREB. Learn Mem 11: 738-747, 2004.

6. Giachino C, De Marchis S, Giampetro C, Parlato R, Perroteau I, Schütz G,Fasolo A and Peretto P: cAMPresponse element-binding protein regulates differentiation and survival of newborn neurons in the olfactory bulb. J Neurosci 25: 10105-10118, 2005.

7. Peltier J, O'Neill A and Schaffer DV: PI3K/Akt and CREB regulate adult neural hippocampal progenitor proliferation and differentiation. Dev Neurobiol 67: 1348-1361, 2007.

8. Sun XM, Lu JH, Qiu YH, Liu Z, Wang XQ and Peng YP: Interleukin- 6 reduces NMDA-induced $\mathrm{Ca}^{2+}$ overload via prevention of $\mathrm{Ca}^{2+}$ release from intracellular store. Int J Neurosci 121: 423-429, 2011.
9. Zhang SJ, Buchthal B and Lau D: A signaling cascade of nuclear calcium-CREB-ATF3 activated by synaptic NMDA receptors defines a gene repression module that protects against extrasynaptic NMDA receptors-induced neuronal cell death and ischemic brain damage. J Neurosci 31: 4978-4990, 2011.

10. Aoki C, Venkatesan C and Go CG: Cellular and subcellular localization of NMDA-R1 subunit immunoreactivity in the visual cortex of adult and neonatal rats. J Neurosci 14: 5202-5222, 1994

11. Yin ZQ, Deng ZM and Crewther SG: Altered expression of alternatively spliced isoforms of the mRNA NMDAR1 receptor in the visual cortex of strabismic cats. Mol Vis 20: 271-276, 2001.

12. Murphy KM, Duffy KR and Jones DG: Experience-dependent changes in NMDAR1 expression in the visual cortex of an animal model for amblyopia. Vis Neurosci 21: 653-670, 2004. 